\title{
THE CONTRIBUTION OF THERMALLY-PULSING ASYMPTOTIC GIANT BRANCH AND RED SUPERGIANT STARS TO THE LUMINOSITIES OF THE MAGELLANIC CLOUDS AT 1-24 $\mu \mathrm{m}$
}

\author{
J. Melbourne ${ }^{1}$ and Martha L. Boyer ${ }^{2,3}$ \\ ${ }^{1}$ Caltech Optical Observatories, Division of Physics, Mathematics and Astronomy, Mail Stop 301-17, California Institute of Technology, \\ Pasadena, CA 91125, USA; jmel@caltech.edu \\ ${ }^{2}$ Observational Cosmology Lab, Code 665, NASA Goddard Space Flight Center, Greenbelt, MD 20771, USA; martha.1.boyer@nasa.gov \\ ${ }^{3}$ Oak Ridge Associated Universities (ORAU), Oak Ridge, TN 37831, USA \\ Received 2012 August 9; accepted 2012 December 12; published 2013 January 22
}

\begin{abstract}
We present the near-through mid-infrared flux contribution of thermally-pulsing asymptotic giant branch (TP-AGB) and massive red supergiant (RSG) stars to the luminosities of the Large and Small Magellanic Clouds (LMC and SMC, respectively). Combined, the peak contribution from these cool evolved stars occurs at $\sim 3-4 \mu \mathrm{m}$, where they produce $32 \%$ of the SMC light, and $25 \%$ of the LMC flux. The TP-AGB star contribution also peaks at $\sim 3-4 \mu \mathrm{m}$ and amounts to $21 \%$ in both galaxies. The contribution from RSG stars peaks at shorter wavelengths, $2.2 \mu \mathrm{m}$, where they provide $11 \%$ of the SMC flux, and 7\% for the LMC. Both TP-AGB and RSG stars are short lived, and thus potentially impose a large stochastic scatter on the near-IR derived mass-to-light $(M / L)$ ratios of galaxies at rest-frame $1-4 \mu \mathrm{m}$. To minimize their impact on stellar mass estimates, one can use the $M / L$ ratio at shorter wavelengths (e.g., at $0.8-1 \mu \mathrm{m})$. At longer wavelengths $(\geqslant 8 \mu \mathrm{m})$, emission from dust in the interstellar medium dominates the flux. In the LMC, which shows strong polycyclic aromatic hydrocarbon (PAH) emission at $8 \mu \mathrm{m}$, TP-AGB and RSG contribute less than $4 \%$ of the $8 \mu \mathrm{m}$ flux. However, $19 \%$ of the SMC $8 \mu \mathrm{m}$ flux is from evolved stars, nearly half of which is produced by the rarest, dustiest, carbon-rich TP-AGB stars. Thus, star formation rates of galaxies, based on an $8 \mu \mathrm{m}$ flux (e.g., observed-frame $24 \mu \mathrm{m}$ at $z=2$ ), may be biased modestly high, especially for galaxies with little PAH emission.
\end{abstract}

Key words: galaxies: fundamental parameters - galaxies: stellar content - stars: AGB and post-AGB

Online-only material: color figures

\section{INTRODUCTION}

Stellar masses of galaxies are typically estimated from a model mass-to-light $(M / L)$ ratio (e.g., Bell \& de Jong 2001), and a measurement of the integrated luminosity at optical or near-infrared wavelengths. Until recently, galaxy $M / L$ ratios were thought to be relatively well behaved, especially at near-infrared wavelengths where the effects of dust and massive main-sequence stars are minimized. However, in the past decade, this assumption has been shown to be flawed because of the influence of short-lived but extremely luminous thermallypulsing asymptotic giant branch (TP-AGB) stars (Maraston et al. 2006; Zibetti et al. 2009, $0.8 \lesssim M \lesssim 8 M_{\odot}$ ) and massive red supergiant stars (RSG; Dalcanton et al. 2012; Melbourne et al. 2012).

Just as the near-IR fluxes of galaxies are often used to estimate their stellar masses, mid-IR fluxes can be used to estimate their star formation rates. For instance, at rest-frame $8 \mu \mathrm{m}$, the flux from starbursts is typically dominated by polycyclic aromatic hydrocarbon (PAH) emission which scales with the star formation rate (SFR; e.g., Díaz-Santos et al. 2008). Likewise, the rest-frame $24 \mu \mathrm{m}$ flux has been shown to scale with the IR luminosity of a galaxy and is therefore used as an SFR indicator (Chary \& Elbaz 2001). However, calibrations of these SFR indicators typically ignore the possibility of stellar contamination in the flux at these wavelengths. Several works have shown that, under certain assumptions, AGB stars could contribute significantly to the mid-IR fluxes of galaxies and therefore affect the SFRs estimated from their mid-infrared fluxes (e.g., Verley et al. 2009; Kelson \& Holden 2010; Chisari $\&$ Kelson 2012). However, as will be discussed in this paper, some of their assumptions may be flawed.
The AGB is a short-lived phase ( $\sim 1$ Myr; e.g., Girardi et al. 2010) near the end of stellar evolution when a star undergoes helium shell burning and swells to a large size, producing a relatively cool photosphere and, potentially, a very infrared (IR) luminous star. Because of the cool temperatures, complex molecules and dust can form in the atmosphere (e.g., Bowen 1988; Winters et al. 2000, 2003; Schirrmacher et al. 2003; Woitke 2006, 2007; Mattsson et al. 2008; van Loon et al. 2008), which in turn can drive strong stellar winds (e.g., Sedlmayr \& Dominik 1995; Elitzur \& Ivezić 2001 and references therein) that ultimately return as much as half of the stellar mass to the interstellar medium. An isolated star will start its AGB phase with an oxygen-rich atmosphere (e.g., O-AGB). However, during the later stages of AGB evolution, a star undergoes a series of thermal-pulses and carbon, dredged up from the interior, pollutes the atmosphere (Iben 1983). When the C/O ratio exceeds unity, the star is classified as a carbon star (e.g., C-AGB), and complex molecules and dust grains form in the escaping winds. These dust grains tend to obscure the star at optical and near-IR wavelengths, but the same dust emits in the mid-IR. Only $20 \%$ of the AGB stars in the LMC/SMC are estimated to be thermally-pulsing (A. G. Bruzual 2012, private communication). However, these TP-AGB stars can be very luminous in the infrared, typically exceeding the luminosity of the tip of the red giant branch (TRGB), sometimes by several magnitudes.

Red supergiants are helium-burning massive stars that form a tight sequence in optical and near-IR color-magnitude diagrams (CMDs; e.g., Figure 1). Stars on this sequence are also termed red helium burning stars (RHeB; Dohm-Palmer \& Skillman 2002; McQuinn et al. 2011; Dalcanton et al. 2012). At the luminous end, RSGs are truly massive, nine solar masses 


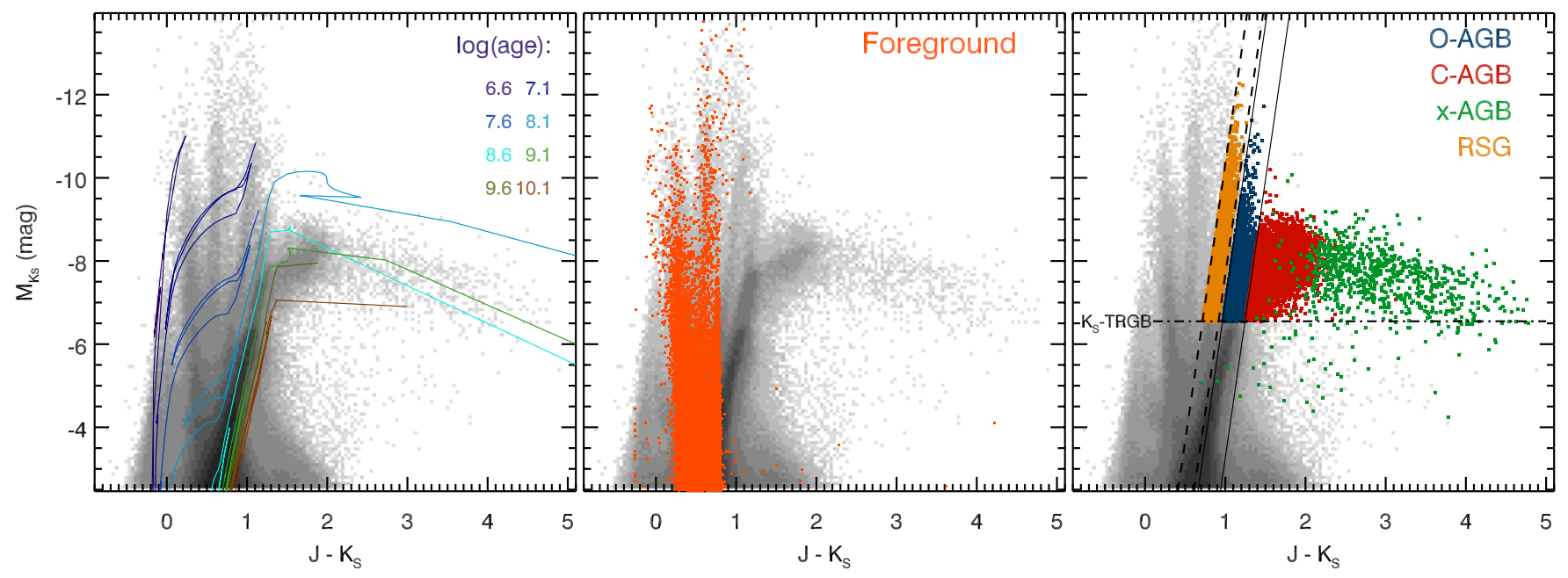

Figure 1. $J-K_{\mathrm{S}}$ vs. $K_{\mathrm{S}}$ CMDs for the LMC (gray density plots). Left panel overlays the Padova isochrones (Bertelli et al. 2008; Marigo et al. 2008; Bertelli et al. 2009; Girardi et al. 2010). Center panel overlays a statistical estimate of the Milky Way foreground stars in the direction of the LMC from TRILEGAL (Girardi et al. 2005). The right panel shows the Boyer et al. (2011) classification of RSG (yellow), O-AGB (blue), and C-AGB (red) stars, with selection regions defined by Cioni et al. (2006). A luminosity cutoff was made at the TRGB, below which the fluxes of these stars become negligible compared with the RGB. The dust enshrouded x-AGB (green) were selected by Boyer et al. (2011) at longer wavelengths; $J-K_{\mathrm{S}}>3.1 \mathrm{mag}$ or [3.6] $-[8]>0.8 \mathrm{mag}$ and [8] $<10 \mathrm{mag}$. Because of their dusty envelopes, the x-AGB stars can be fainter than the $K_{\mathrm{S}}$-band TRGB and yet be significantly brighter than the TRGB at longer wavelengths.

(A color version of this figure is available in the online journal.)

or more, and thus were born in very recent star formation episodes (e.g., $<0.1 \mathrm{Gyr}$ ). At the luminosity of the $K$-band TRGB, the Padova isochrones predict more modest masses, 6-7 $M_{\odot}$ (Bertelli et al. 2008, 2009; Marigo et al. 2008; Girardi et al. 2010), and in fact the RHeB sequence continues to lower luminosities for stars of even smaller mass (e.g., McQuinn et al. 2011).

Combined, TP-AGB and RSG stars have been shown to account for as much $40 \%$ of the integrated $1.6 \mu \mathrm{m}$ flux, even in local galaxies, where the red giant branch (RGB) is well developed (Melbourne et al. 2012). In the early universe, when the RGB has not had time to form, the TP-AGB and RSG stars are expected to contribute even larger fractions (as much as 70\%) of the near-IR light, even though they represent a tiny fraction of the stellar mass (Maraston et al. 2006; Melbourne et al. 2012). Thus, these stars must be accounted for to accurately model the infrared $M / L$ ratios of galaxies (Zibetti et al. 2009; Ilbert et al. 2010).

Not only do these stars contribute to the near-IR luminosities of galaxies, but they also can be significant sources of mid-IR light (e.g., Boyer et al. 2011). TP-AGB stars, in particular, can produce significant amounts of warm dust (e.g., Bowen 1988; Schirrmacher et al. 2003).

These dusty shells both emit in the mid-infrared and obscure the TP-AGB stars at optical wavelengths (e.g., Reid 1991).

The flux contribution of TP-AGB and RSG stars has now been well quantified at 0.8 and $1.6 \mu \mathrm{m}$ in a set of 23 local dwarfs and spirals (Girardi et al. 2010; Melbourne et al. 2012). Similarly, the 3.6 and $4.5 \mu \mathrm{m}$ flux contribution of the most extreme (dusty) TP-AGB stars has been quantified in a set of six nearby galaxies (Gerke \& Kochanek 2013). Likewise, the mid-IR $(8-24 \mu \mathrm{m})$ flux contribution from carbon stars has been estimated from stellar population synthesis models of SINGS galaxies (Chisari \& Kelson 2012). However, there has not been a study that quantifies the flux contributions of these stars across the full 1-24 $\mu \mathrm{m}$ wavelength range for a galaxy where complete samples of TP-AGB and RSG stars are actually resolved and individually measured.
In this paper, we use complete samples of TP-AGB and massive RSG stars in the Magellanic Clouds from the Spitzer legacy program "Surveying the Agents of Galaxy Evolution" (SAGE; Blum et al. 2006; Meixner et al. 2006; Gordon et al. 2011; Boyer et al. 2011). We build off of the results of Boyer et al. (2011), who showed that the TP-AGB and RSG stars contribute significantly to the near- and mid-IR point-source fluxes in these galaxies. We now show that their contribution to the total (stellar and interstellar) fluxes of the SMC and LMC varies significantly from 1 to $24 \mu \mathrm{m}$. We also find significant differences between our measured flux contributions and those from several recent studies in the literature. We find a significantly larger TP-AGB contribution at $3.6 \mu \mathrm{m}$ than is found in Gerke \& Kochanek (2013) and Meidt et al. (2012a). Conversely, we find that the 8-24 $\mu \mathrm{m}$ AGB flux contributions estimated in Kelson \& Holden (2010) and Chisari \& Kelson (2012) are likely significantly overestimated. We discuss the implications of these results for estimates of stellar mass and SFRs of galaxies.

\section{THE DATA}

This paper uses several types of observations: (1) groundbased, seeing-limited near-infrared imaging to measure the nearinfrared fluxes of TP-AGB and RSG stars in the Magellanic Clouds, (2) space-based, low-resolution near-infrared imaging to determine the total integrated near-infrared luminosities of the Magellanic Clouds, and (3) space-based, mid-infrared imaging to determine the mid-infrared fluxes of both the stars and the integrated galaxy light. In addition to the IR data, we use groundbased optical data to explore the flux contribution of short-lived massive main-sequence stars at shorter wavelengths. Details of these data sets are provided below. All magnitudes are reported in the Vega system.

\subsection{Stellar Photometry}

Multi-wavelength stellar photometry of the stars in the Large and Small Magellanic Clouds (LMC and SMC, respectively) was compiled by the SAGE team. $J$ and $K_{\mathrm{S}}$ (i.e., 1.2 and 
$2.2 \mu \mathrm{m})$ near-infrared photometry was obtained from the Two Micron All Sky Survey (2MASS; Skrutskie et al. 2006). Midinfrared photometry at $3.6,4.5,5.8$, and $8 \mu \mathrm{m}$ was obtained with the Spitzer Space Telescope Infrared Array Camera (IRAC; Fazio et al. 2004). Photometry at $24 \mu \mathrm{m}$ was obtained with the Multiband Imaging Photometer for Spitzer (MIPS; Rieke et al. 2004). A discussion of the Spitzer observations, image reductions, photometry, and source matching for the SMC is given in Gordon et al. (2011), and the LMC photometry is discussed in Meixner et al. (2006). Optical, BVI, data were compiled by the Magellanic Clouds Photometric Survey (MCPS; Zaritsky et al. 2002).

Stellar classifications for stars in the SAGE data were presented in Boyer et al. (2011). Here, we briefly summarize the method; for a full description of the stellar classification and sources of possible contamination, see Boyer et al. (2011). Classification was carried out by examining the locations of stars in multi-wavelength color-magnitude space. Less-dust-obscured TP-AGB and RSG stars were identified in near-infrared CMDs, where they are among the most luminous stars, e.g., above the $K_{\mathrm{S}}$-band TRGB. At these wavelengths, sequences of carbonrich and oxygen-rich AGB stars (C-AGB and O-AGB stars, respectively) separate by $J-K_{\mathrm{S}}$ color, with C-AGB stars typically being redder than O-AGB stars. Likewise, RSG stars form a tight sequence slightly blueward of the O-AGB stars. These sequences are clearly visible in the $J-K_{\mathrm{S}} \mathrm{CMD}$ of the LMC shown in Figure 1 which also shows the color-magnitude boundaries used to identify these different sequences (adopted from Cioni et al. 2006). While these boundaries are useful for identifying different classes of stars, we note that there will be some overlap between the populations. Boyer et al. (2011) provide a detailed discussion of classifications including estimates of the numbers of misclassified stars, which are small. We note that the anomalous O-rich AGB stars reported by Boyer et al. (2011) are included with the O-AGB sample here.

The most dust enshrouded TP-AGB stars are significantly extinguished even in the near-IR, and therefore they must be identified at longer wavelengths (e.g., Reid 1991). Boyer et al. (2009) found that short wavelength searches can miss up to $40 \%$ of the TP-AGB stars. These so-called extreme AGB stars (x-AGB) were identified by their colors at mid-IR wavelengths (3.6-8 $\mu \mathrm{m}$ ). Most $\mathrm{x}$-AGB stars are C-rich (van Loon et al. 2005). Combining these two identification methods allows for the selection of near-complete samples of TP-AGB stars (e.g., these classifications do not miss the most dust enshrouded stars, which can be missed by classifications that rely entirely on shorter wavelengths).

While these techniques will recover the bulk of the luminous TP-AGB stars, some TP-AGB stars will be missed because they are at a minimum in their thermal pulsation cycle and are fainter than the TRGB. Models of the TP-AGB stars in the Magellanic Clouds based on the Padova stellar evolution tracks reveal that $<10 \%$ of them lie below the TRGB due to the thermal pulse cycle (e.g., Marigo \& Girardi 2007). We have included these subluminous TP-AGB stars in the uncertainties of the integrated TP-AGB fluxes by assuming that they have the luminosity distribution of the top magnitude of the RGB. We added this uncertainty in quadrature to the other sources of uncertainty, increasing the upper error bars in Table 1 and Figure 2 by $<3 \%$.

We also note that there are AGB stars that are not thermallypulsing. These are lower-luminosity AGB stars and also lie below the TRGB. These stars cannot be easily selected in CMD space because they overlap the RGB stars. However, as these AGB stars are also relatively rare compared with the equally luminous RGB, they will not contribute significantly to the total flux of the galaxy.

The total numbers of SMC and LMC stars within each stellar classification bin are presented in Table 1.

\subsection{Foreground Contamination}

The CMDs of the LMC and SMC also contain some Milky Way foreground stars with the colors and luminosities of Magellanic Cloud AGB and RSG stars. We applied a statistical correction for foreground contamination of Milky Way stars at each wavelength. We used TRILEGAL (Girardi et al. 2005) to model the numbers and fluxes of Milky Way stars in the direction of the Magellanic Clouds in the same filters as our data. The TRILEGAL photometry was then run through the same classification scheme as the RSG and TP-AGB stars. The contamination is largest for the RSG population of the SMC $(\sim 25 \%)$. In the LMC, the RSG contamination is negligible because the higher metallicity of the LMC causes all of the RSG and AGB stars to be redder than they are in the SMC, resulting in less overlap with the foreground sources in the near-IR CMD (see Figure 1). Likewise, the AGB populations in both galaxies are effectively foreground contamination free. The estimated numbers of foreground stars within each stellar class are also presented in Table 1.

Note: stars in the Milky Way foreground clusters 47 Tuc and NGC 362, in the direction of the SMC, were removed from the data (see Boyer et al. 2011).

\subsection{Integrated Galaxy Photometry}

Measuring the total luminosity of the Magellanic Clouds is challenging because of their very large areas on the sky. Groundbased, near-IR images are dominated by thermal background flux, and thus accurately discerning sky from unresolved galaxy becomes nearly impossible. Fortunately, these issues are largely eliminated by going to space, where the IR thermal background is minimal and $C O B E / D I R B E$ (Hauser et al. 1998) provides a large beam on the sky (nearly a degree).

We use the $C O B E$ /DIRBE integrated flux density estimates of Israel et al. (2010) for the Magellanic clouds at 1.25, 2.2, and $3.5 \mu \mathrm{m}$. The SMC flux is measured within a $12.3 \times 12.3$ area, and the LMC flux covers a $15.3 \times 15.3$ area. These measurements were carefully corrected for foreground (and background) contamination by Israel et al. (2010). The Israel et al. flux density estimates for the Clouds are presented here in Table 1.

Similarly, the global SMC and LMC IR fluxes from the Spitzer data at 3.6, 4.5, 5.8, 8, and $24 \mu \mathrm{m}$ were made by Gordon et al. (2011) and K. D. Gordon et al. (in preparation). These fluxes were measured within circular apertures of radius 2.25 for the SMC and 3.75 for the LMC. For both galaxies, a sky annulus with a 0.2 width was used to estimate and subtract the background/foreground. To within the uncertainties, the integrated DIRBE fluxes at $3.5 \mu \mathrm{m}$ from Israel et al. match the Spitzer fluxes at $3.6 \mu \mathrm{m}$ from Gordon et al. The Spitzer fluxes are provided in Table 1.

The spectral energy distributions (SEDs) of the SMC and LMC are shown in Figure 2. Also overlaid are dust model predictions from Bot et al. (2010). While the stellar flux is declining with wavelength, the warm dust and PAH components are increasing. 
Table 1

Integrated Flux Density of the SMC and LMC from 1 to $24 \mu \mathrm{m}$ and Percentage of that Flux Contributed by TP-AGB and RSG Stars

\begin{tabular}{|c|c|c|c|c|c|c|c|c|c|c|}
\hline Type & Stars $^{\mathrm{a}}$ & Fgnd $^{b}$ & $1.2 \mu \mathrm{m}^{\mathrm{c}}$ & $2.2 \mu \mathrm{m}^{\mathrm{c}}$ & $3.5 \mu \mathrm{m}^{\mathrm{d}}$ & $3.6 \mu \mathrm{m}^{\mathrm{e}}$ & $4.5 \mu \mathrm{m}^{\mathrm{e}}$ & $5.8 \mu \mathrm{m}^{\mathrm{e}}$ & $8 \mu \mathrm{m}^{\mathrm{e}}$ & $24 \mu \mathrm{m}^{\mathrm{e}}$ \\
\hline \multicolumn{11}{|l|}{ SMC } \\
\hline & & & \multicolumn{8}{|c|}{ Integrated Flux (Jy) } \\
\hline & & & $670 \pm 80^{f}$ & $525 \pm 65^{\mathrm{f}}$ & $280 \pm 40^{\mathrm{f}}$ & $300 \pm 20^{\mathrm{g}}$ & $220 \pm 10^{\mathrm{g}}$ & $220 \pm 10^{\mathrm{g}}$ & $200 \pm 10^{g}$ & $350 \pm 10^{g}$ \\
\hline & & & \multicolumn{8}{|c|}{ Fractional Contribution $(\%)$} \\
\hline O-AGB & 3353 & 4 & $5.7_{-0.7}^{+1.1}$ & $8.4_{-1.0}^{+1.5}$ & $8.0_{-1.1}^{+1.5}$ & $7.5_{-0.5}^{+1.1}$ & $6.0_{-0.3}^{+0.8}$ & $4.5_{-0.2}^{+0.6}$ & $3.1_{-0.2}^{+0.4}$ & $0.14_{-0.01}^{+0.01}$ \\
\hline C-AGB & 1597 & $<1$ & $3.0_{-0.4}^{+0.4}$ & $6.6_{-0.8}^{+0.9}$ & $9.1_{-1.3}^{+1.4}$ & $8.5_{-0.6}^{+0.8}$ & $7.1_{-0.3}^{+0.6}$ & $5.3_{-0.2}^{+0.4}$ & $4.8_{-0.2}^{+0.4}$ & $0.31_{-0.01}^{+0.02}$ \\
\hline $\mathrm{x}$-AGB & 309 & $<1$ & $0.2_{-0.1}^{+0.1}$ & $1.2_{-0.1}^{+0.1}$ & $4.8_{-0.7}^{+0.7}$ & $4.5_{-0.3}^{+0.3}$ & $6.5_{-0.3}^{+0.3}$ & $6.9_{-0.3}^{+0.3}$ & $7.6_{-0.4}^{+0.4}$ & $1.30_{-0.04}^{+0.04}$ \\
\hline RSG & 2340 & 598 & $8.9_{-1.1}^{+1.1}$ & $10.9_{-1.4}^{+1.4}$ & $10.0_{-1.4}^{+1.4}$ & $9.4_{-0.6}^{+0.6}$ & $7.6_{-0.3}^{+0.3}$ & $5.5_{-0.3}^{+0.3}$ & $3.7_{-0.2}^{+0.2}$ & $0.33_{-0.01}^{+0.01}$ \\
\hline Total & 7599 & 602 & $17.8_{-1.3}^{+1.8}$ & $27.1_{-1.9}^{+2.7}$ & $31.9_{-2.3}^{+3.4}$ & $29.8_{-1.0}^{+2.5}$ & $27.2_{-0.6}^{+2.3}$ & $22.3_{-0.5}^{+2.0}$ & $19.3_{-0.5}^{+2.1}$ & $2.1_{-0.1}^{+0.2}$ \\
\hline
\end{tabular}

LMC

Integrated Flux (Jy)

$4520 \pm 650^{\mathrm{f}} 3770 \pm 540^{\mathrm{f}} 2190 \pm 300^{\mathrm{f}} 2080 \pm 100^{\mathrm{h}} 1350 \pm 70^{\mathrm{h}} 1850 \pm 200^{\mathrm{h}} 5980 \pm 300^{\mathrm{h}} 7640 \pm 200^{\mathrm{h}}$ Fractional Contribution (\%)

\begin{tabular}{lcccccccccc}
\cline { 3 - 10 } O-AGB & 15635 & $<1$ & $6.8_{-1.0}^{+2.6}$ & $9.9_{-1.1}^{+3.1}$ & $8.8_{-0.8}^{+3.1}$ & $9.3_{-0.5}^{+3.2}$ & $8.3_{-0.4}^{+3.1}$ & $4.6_{-0.5}^{+1.7}$ & $0.9_{-0.1}^{+0.3}$ & $0.11_{-0.01}^{+0.22}$ \\
C-AGB & 5628 & $<1$ & $2.6_{-0.4}^{+0.4}$ & $6.0_{-0.6}^{+0.7}$ & $7.6_{-0.7}^{+0.7}$ & $8.1_{-0.4}^{+0.5}$ & $7.3_{-0.4}^{+0.4}$ & $3.7_{-0.4}^{+0.4}$ & $1.0_{-0.1}^{+0.1}$ & $0.11_{-0.01}^{+0.01}$ \\
x-AGB & 989 & $<1$ & $0.1_{-0.1}^{+0.1}$ & $0.7_{-0.1}^{+0.1}$ & $2.9_{-0.3}^{+0.3}$ & $3.0_{-0.1}^{+0.2}$ & $5.2_{-0.3}^{+0.3}$ & $4.3_{-0.5}^{+0.5}$ & $1.4_{-0.1}^{+0.1}$ & $0.35_{-0.01}^{+0.01}$ \\
RSG & 4361 & 13 & $5.5_{-0.8}^{+0.8}$ & $6.9_{-0.7}^{+0.7}$ & $6.0_{-0.5}^{+0.5}$ & $6.3_{-0.3}^{+0.3}$ & $5.5_{-0.3}^{+0.3}$ & $3.0_{-0.3}^{+0.3}$ & $0.6_{-0.1}^{+0.1}$ & $0.16_{-0.01}^{+0.01}$ \\
\hline Total & 26613 & 13 & $15.1_{-1.3}^{+2.8}$ & $23.5_{-1.4}^{+3.3}$ & $25.2_{-1.2}^{+3.3}$ & $26.6_{-0.7}^{+3.3}$ & $26.3_{-0.7}^{+3.2}$ & $15.6_{-0.9}^{+1.9}$ & $3.9_{-0.1}^{+0.4}$ & $0.7_{-0.1}^{+0.2}$ \\
\hline
\end{tabular}

Notes.

${ }^{\text {a }}$ Number of stars of given type detected at $3.6 \mu \mathrm{m}$; the number varies slightly with wavelength.

${ }^{b}$ Number of Milky Way foreground stars as estimated by TRILEGAL (Girardi et al. 2005).

${ }^{\mathrm{c}}$ Integrated Flux from COBE/DIRBE (at 1.25 and $2.2 \mu \mathrm{m}$ ), Stellar Flux from 2MASS (at 1.2 and $2.2 \mu \mathrm{m}$ ).

${ }^{\mathrm{d}}$ Integrated Flux from COBE/DIRBE (at $3.5 \mu \mathrm{m}$ ), Stellar Flux from Spitzer (at $3.6 \mu \mathrm{m}$ ).

${ }^{\mathrm{e}}$ Integrated Flux from Spitzer, Stellar Flux from Spitzer.

${ }^{\mathrm{f}}$ From Israel et al. (2010).

g From Gordon et al. (2011).

${ }^{\mathrm{h}}$ From K. D. Gordon et al. (in preparation).

We compare these integrated galaxy fluxes gathered from the literature to the fluxes from the TP-AGB and RSG stars as measured from 2MASS and Spitzer photometry. The 2MASS $J$ and $K_{\mathrm{S}}$ bands are similar to the $C O B E / \mathrm{DIRBE} 1.25$ and $2.2 \mu \mathrm{m}$ filters, however, the DIRBE filters are $0.05 \mu \mathrm{m}(15 \%)$ wider at the long wavelength end. Similarly, the COBE/DIRBE $3.5 \mu \mathrm{m}$ filter is slightly bluer and about $25 \%$ wider than the Spitzer $3.6 \mu \mathrm{m}$ filter. These slight filter mismatches may increase the uncertainties on the measured AGB and RSG flux fractions by modest amounts. There are no filter mismatches for the 3.6-24 $\mu \mathrm{m}$ flux ratios, as all measurements are made from the same Spitzer images.

\subsection{Uncertainties}

In the following section, we report the fractional flux contribution of TP-AGB and RHeB stars to the Magellanic Clouds as given by:

$$
\text { Flux Fraction }=\frac{\text { Stellar Flux }- \text { Foreground Stellar Flux }}{\text { Integrated Galaxy Flux }} .
$$

The uncertainties for these measurements are constructed from the following components. First, we assume the published uncertainties for the integrated fluxes of the Magellanic Clouds at each wavelength. Second, we compile the photometric uncertainty in the integrated stellar flux from the photometric uncertainty of each star added in quadrature. Third, we estimate the uncertainty on the foreground correction by taking the Poisson uncertainty on the numbers of foreground stars in each classification multiplied by the typical flux of those stars. We propagate these uncertainties through Equation 1. Fourth, we include an estimate of the uncertainty introduced by AGB stars that are fainter than the TRGB as described in Section 2.1.

Finally, we have quantified how much of the TP-AGB and RSG population we have missed due to limited spatial coverage within the apertures we used to measure each galaxy's integrated flux. In both galaxies, the TP-AGB and RSG stars follow a spherical distribution centered on the Bars (e.g., Blum et al. 2006; Boyer et al. 2011). For the SMC, the SAGE footprint includes an additional strip of coverage that extends $>6$ deg along the SMC Tail, and for the LMC, the SAGE coverage stretches along the Disk, $>4.5 \mathrm{deg}$ from the Bar's center. We can use this extended coverage to measure the radial profile of AGB stars in the Bars by counting the number of AGB stars in concentric ellipses centered on the Bars. We find that the AGB population is rather tightly concentrated, dropping to very small numbers by about 2 deg (along the minor axes) from the population centers. By extrapolating these radial profiles in all directions, we estimate that we have missed about 500 SMC AGB stars and 900 LMC AGB stars (10 SMC RSGs and 20 LMC RSGs) by restricting our apertures to 2.25 and $3.75 \mathrm{deg}$ for the SMC and LMC, respectively. Assuming that the missed stars have a flux distribution that is similar to the measured TP-AGB/RSG population, this amounts to an extra $<1 \%$ uncertainty on the integrated AGB (and RSG) flux, which we have included in the upper uncertainties in Table 1 and Figure 2. 


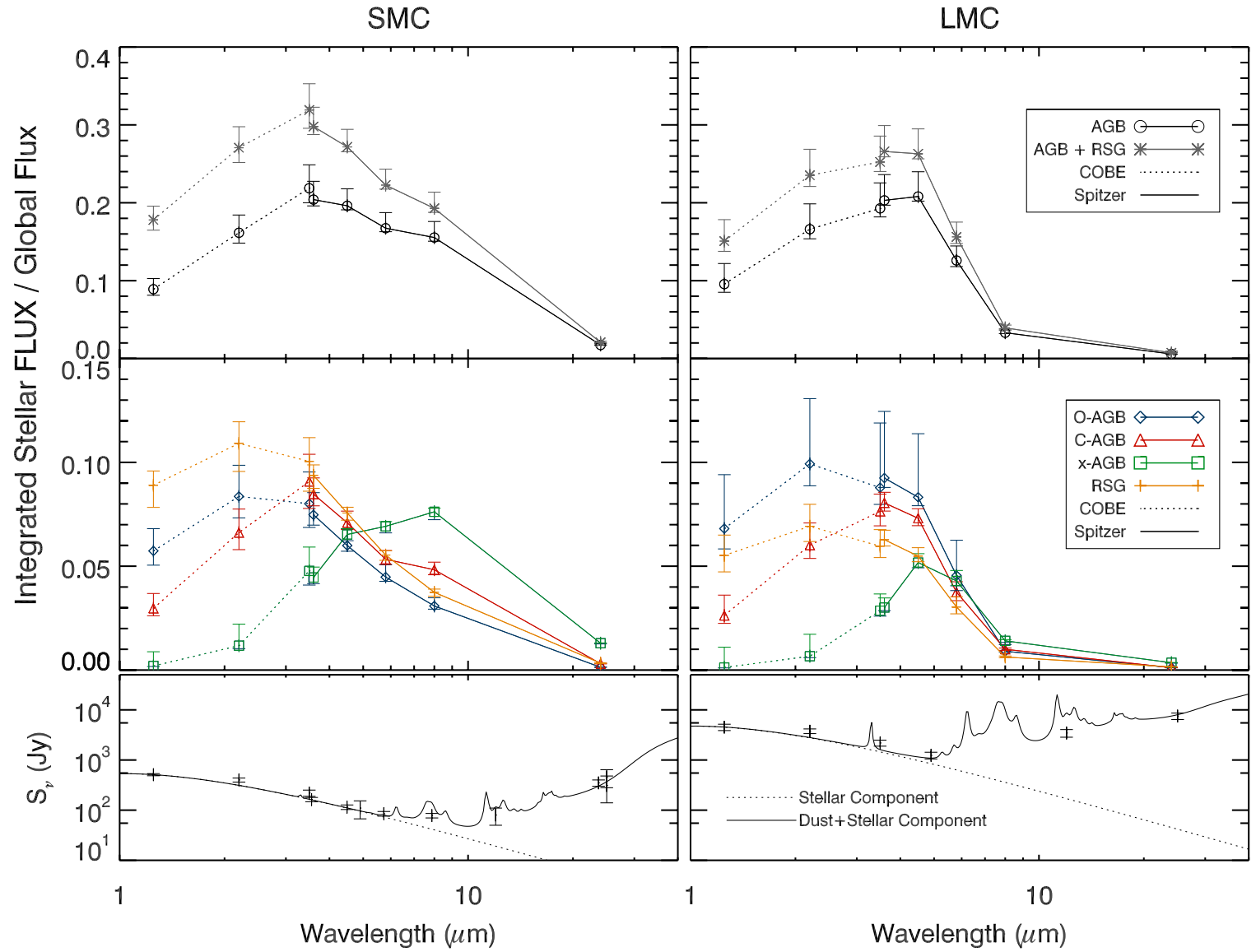

Figure 2. Fractional contribution from TP-AGB stars (open symbols) and RSG stars (plus symbols) to the integrated near- and mid-IR fluxes of the SMC and LMC (top and middle panels). The contributions divided by subspecies are given in the middle panels, while the total contributions from all TP-AGB stars (circles) and TP-AGB + RSG (asterisk) are shown in the top panels. The bottom panel shows the integrated SEDs of the LMC and SMC and a model of the dust emission from Bot et al. (2010). While the stellar flux declines with wavelength (dotted line), the dust emission increases. The flux contribution of TP-AGB stars peaks at $\sim 3-4 \mu \mathrm{m}$. The contribution from RSG stars peaks at shorter wavelengths (roughly $2.2 \mu \mathrm{m}$ ). Combined, these evolved stars contribute $32 \%$ of the SMC light at 3-4 $\mu \mathrm{m}$ and $19 \%$ at $8 \mu \mathrm{m}$. The flux contributions of the TP-AGB and RSG at $24 \mu \mathrm{m}$ are small, $<3 \%$, in both galaxies.

(A color version of this figure is available in the online journal.)

\section{RESULTS: THE MULTI-WAVELENGTH CONTRIBUTION OF TP-AGB AND RSG STARS TO THE INFRARED FLUX OF THE MAGELLANIC CLOUDS}

Figure 2 shows the fraction of the integrated galaxy light produced by TP-AGB (open symbols) and RSG (plus symbols) stars in the SMC and LMC as a function of wavelength from 1 to $24 \mu \mathrm{m}$. These results are also presented in Table 1. At $3.6 \mu \mathrm{m}$, these rare stars contribute over $30 \%$ of the SMC flux, even though they represent a negligible fraction of the stellar mass of the galaxy (numbers of stars of each type are also shown in Table 1). In the LMC, they contribute more than a quarter of the $3.6 \mu \mathrm{m}$ light. At $24 \mu \mathrm{m}$, the stellar contribution to the total luminosity is $<3 \%$ for both galaxies.

The flux contribution from RSG stars, which are bluer than AGB stars, peaks near $\sim 2 \mu \mathrm{m}$. In the SMC, they are responsible for over $10 \%$ of the $K$-band light. In the LMC, the fraction is lower, 7\%. Part of this difference is no doubt due to the detailed star formation histories of these two galaxies over the last $0.1 \mathrm{Gyr}$, with the SMC having a higher specific star formation rate over that period (e.g., Harris \& Zaritsky 2004, 2009).

The flux contribution from TP-AGB stars peaks in the Spitzer IRAC bands between 3 and $4 \mu \mathrm{m}$, reaching $15 \%-20 \%$ for both the LMC and SMC. The similarity of the results for the two galaxies suggests that over the longer timescale of 0.1-2 Gyr, these galaxies have had similar star formation histories, as has been inferred by studies of their CMDs (Harris \& Zaritsky 2004, 2009).

Figure 2 also shows the TP-AGB populations subdivided into the three categories previously discussed: O-AGB, C-AGB, and $\mathrm{x}$-AGB. Despite the fact that the O-AGB population outnumbers the C-AGB population by a factor of 1.8 in the SMC and 2.4 in the LMC, the contribution to the global flux of the O-AGB and C-AGB populations are similar at $\lambda>3 \mu \mathrm{m}$, with each contributing roughly equal parts to the total TP-AGB flux. However, the contribution from O-AGB stars peaks at shorter wavelengths (near $2.2 \mu \mathrm{m}$ ) than the C-AGB star contribution (near $3.6 \mu \mathrm{m}$ ). In contrast, the $\mathrm{x}$-AGB stars account for less than $1 \%$ of the global flux at $\lambda<2 \mu \mathrm{m}$, but exceed the flux contribution of the other two TP-AGB types at $\lambda>4 \mu \mathrm{m}$. This is true even though the $\mathrm{x}$-AGB population is numerically tiny, comprising $<4 \%$ of the total number of TP-AGB stars in both galaxies.

\section{DISCUSSION}

We present, for the first time, the near- through mid-IR flux contributions of complete samples of TP-AGB and RSG stars to the integrated luminosities of the Magellanic Clouds. We find 
that these stars can contribute non-negligible flux fractions from 1 to $8 \mu \mathrm{m}$ (e.g., $15 \%-30 \%$ ), and smaller fractions at $24 \mu \mathrm{m}$ (e.g., $<3 \%$ ). Below, we compare these results to previous work and discuss the implications for estimating stellar masses and star formation rates of galaxies.

\subsection{The Importance of TP-AGB and RSG Stars at Near-IR Wavelengths}

Rest-frame near-IR fluxes typically anchor the stellar mass estimates of galaxies (e.g., Maraston et al. 2006; Conroy et al. 2009; Zibetti et al. 2009; Eskew et al. 2012). At these wavelengths, dust obscuration is significantly reduced compared with optical light, and a galaxy's near-IR luminosity is much less affected by the short-lived O- and B-type stars produced by current star formation. Instead, the near-IR is typically thought to be dominated by older, easily modeled RGB stars.

However, we have shown that TP-AGB and massive RSG stars can also account for large fractions of the near-IR light (e.g., $>30 \%$ at $3.6 \mu \mathrm{m}$ for the SMC), even though they contribute essentially nothing to the total stellar masses of galaxies. A galaxy with the same $3.6 \mu \mathrm{m}$ luminosity as the SMC but comprised of entirely old stellar populations (e.g., $>3 \mathrm{Gyr}$ ) must be nearly twice as massive as the SMC to make up for the missing stellar luminosity provided by the TP-AGB and RSG at that wavelength. Thus, a galaxy's flux at 3-4 $\mu \mathrm{m}$ (and hence its $M / L$ ratio) is highly sensitive to the star formation history.

The large TP-AGB and RSG near-IR flux contributions we measure for the Magellanic Clouds fit well with the contributions reported in Melbourne et al. (2012) for a sample of 23 nearby dwarfs and small spirals, which ranged from 5\% to $40 \%$ at $1.6 \mu \mathrm{m}$. Similarly, our results are well matched to the predictions of Bruzual (2011), which discussed the contribution of AGB stars to the $K$-band flux of their stellar population synthesis models. Their results for a galaxy with $Z=0.008$ and a declining SFR with an $e$-folding time of $1 \mathrm{Gyr}$ are consistent with what we see in the Magellanic Clouds. These large flux contributions suggest that galaxies with even modest populations of TP-AGB and RSG stars will have very different near-IR $M / L$ ratios than galaxies lacking these populations, making near-IR based stellar mass estimates difficult.

However, some authors find significantly smaller near-IR flux contributions from TP-AGB stars. For instance, Gerke \& Kochanek (2013) calculate a smaller $3.6 \mu \mathrm{m}$ flux contribution from the TP-AGB in the Magellanic clouds, only $5 \%$ of the integrated light. They selected AGB stars using the [3.6] - [4.5] color, however, they selected a CMD region that is comprised predominantly of $\mathrm{x}$-AGB stars. Thus, they miss the bulk of the TP-AGB altogether. The top panel of Figure 3 shows the distribution of [3.6] - [4.5] colors of TP-AGB stars in the LMC. The Gerke \& Kochanek (2013) sample has a mean color similar to the X-AGB alone (green dot-dashed line), which are significantly redder than the overall sample (dotted line). If we compare only the $\mathrm{x}$-AGB stars in our sample to their result, then we find a similarly small contribution of $\sim 3 \%$. Including the full TP-AGB sample results in a much higher flux contribution at $3.6 \mu \mathrm{m}$, as discussed above.

Meidt et al. (2012a) show a similar result to Gerke \& Kochanek (2013), only they used unresolved Spitzer 3.6 and $4.5 \mu \mathrm{m}$ observations of galaxies in the Spitzer Survey of Stellar Structure in Galaxies ( $S^{4} G$; Sheth et al. 2010). Their work spatially separates (on a pixel-by-pixel basis) older stellar populations from warm dust, PAH emission, and evolved stars, based on the colors of regions in the Spitzer data. Thus, they
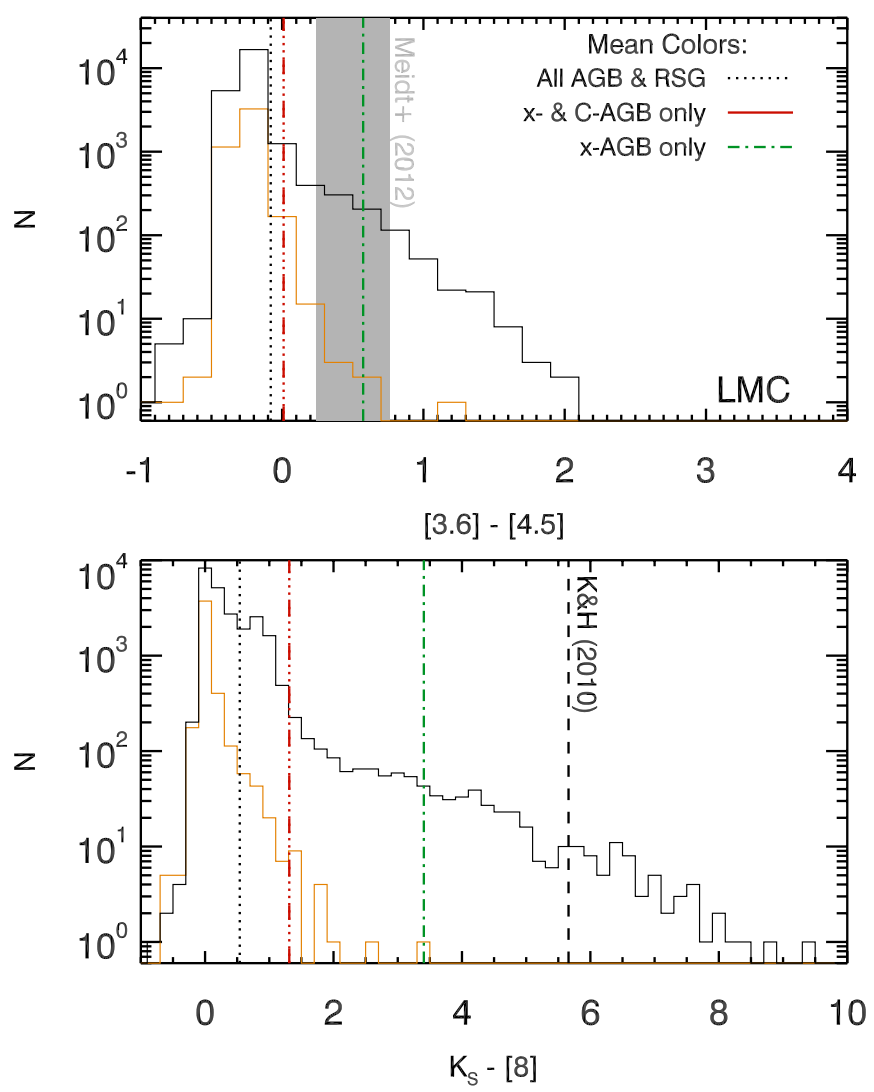

Figure 3. Histograms of the [3.6] - [4.5] (top) and $K_{\mathrm{S}}-$ [8] (bottom) colors of the AGB (black) and RSG (yellow) stars in the LMC. Also shown are various color selections used in the literature to select AGB populations. The top panel shows the [3.6] - [4.5] color range used by Meidt et al. (2012b; shaded region) to isolate AGB and RSG stars, which roughly matches the mean selection used in Gerke \& Kochanek (2013). Both of these selections are significantly redder than the mean AGB and RSG colors in the LMC (dotted line; and the SMC, see Boyer et al. 2011). The lower panel shows the typical carbon star color used in the Kelson \& Holden (2010) models (dashed line), which is significantly redder than the mean color for the X-AGB stars (green dash-dotted line) and over 4 mag redder than the mean carbon star color (red triple-dot-dashed line).

(A color version of this figure is available in the online journal.)

are able to nicely constrain the warm dust and PAH emission to roughly $5 \%-15 \%$ of the $3.6 \mu \mathrm{m}$ flux in a sample of six nearby galaxies. Likewise, they find that pixels dominated by evolved AGB and RSG stars account for only another roughly $5 \%$ of the $3.6 \mu \mathrm{m}$ light. However, as with the Gerke \& Kochanek (2013) result, their assumed AGB color of [3.6] - [4.5] = 0.24-0.76 (gray shaded region of Figure 3 ) is significantly redder than the bulk of the TP-AGB stars in the LMC which have a mean color of [3.6] $-[4.5]=0.0$ (red triple-dot-dashed line). In fact, the mean [3.6] - [4.5] color for TP-AGB and RSG in the LMC is $[3.6]-[4.5]=-0.07$ (dotted line), exactly the color Meidt et al. (2012a) assign to old stars. Unfortunately, that means that Meidt et al. (2012a) have only selected the reddest TP-AGB stars and, as a result, they are likely underestimating the contribution of TP-AGB stars at $3.6 \mu \mathrm{m}$.

The results we have presented here suggest that the near-IR may not be optimized for stellar mass estimates of galaxies. The $3.6 \mu \mathrm{m}$ band, in particular, is the most problematic for the effects of short-lived TP-AGB and RSG stars, which can impose a stochastic variability on the near-IR $M / L$ ratio of stellar populations (e.g., Melbourne et al. 2012). However, it should be possible to minimize the effects of the TP-AGB and RSG stars on estimates of the near-IR $M / L$ ratios of galaxies by using bluer 


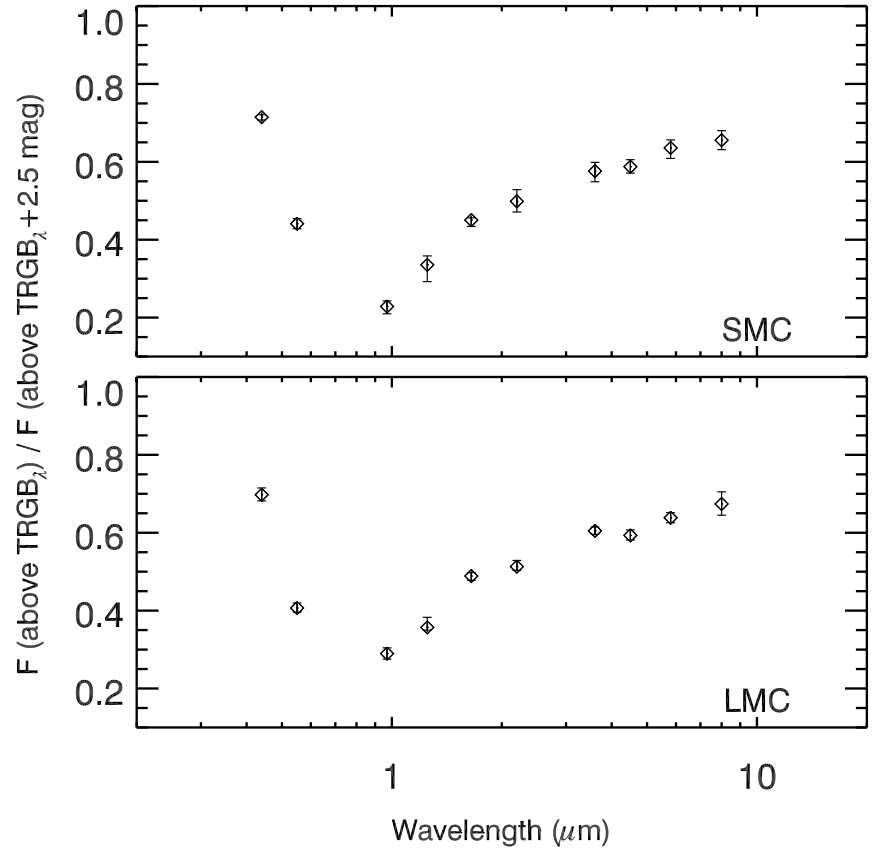

Figure 4. Contribution of luminous stars (with fluxes $>$ than the TRGB) to the total point-source flux (measured to $2.5 \mathrm{mag}$ below the TRGB) as a function of wavelength. At the blue end the $\mathrm{O}$ and $\mathrm{B}$ stars contribute significantly to the point-source flux. At the red end, RSG and TP-AGB stars contribute significantly to the total. As all of these stars are short lived, they will result in a rapidly changing $M / L$ ratio at these wavelengths. The impact of luminous short-lived stars on the stellar mass estimates of galaxies can be minimized by using the $M / L$ ratio at $0.8-1.0 \mu \mathrm{m}$, where this plot shows a minimum.

wavebands. The TP-AGB and RSGs flux contribution at $1 \mu \mathrm{m}$ is roughly half that of the 3.6- $\mu \mathrm{m}$ flux contribution. Of course, bluer wavelengths will be more impacted by extinction from dust and emission from hot $\mathrm{O}$ and B stars, but the $I$ or $J$ bands may prove to be the ideal compromise. To test this hypothesis, we compare the point-source flux above the TRGB to the total point-source flux (which is measurable to $2.5 \mathrm{mag}$ below the TRGB) as a function of wavelength from 0.5 to $3.6 \mu \mathrm{m}$. Figure 4 displays the results, and shows that there is indeed a minimum flux contribution from luminous short-lived stars as a function of wavelength. The minimum occurs between 0.8 and $1 \mu \mathrm{m}$. At shorter wavelengths, massive main-sequence $\mathrm{O}$ and $\mathrm{B}$ stars contribute large fractions of light. At longer wavelengths, the TP-AGB and RSG stars contribute large fractions. This plot suggests that rest-frame $I$ through $J$ bands will indeed provide $M / L$ ratios that are the most stable against stellar population history and the influence of these short-lived luminous stars.

\subsection{The Importance of TP-AGB and RSG Stars at Mid-IR Wavelengths}

Because mid-IR light is produced by warm dust and PAH emission in star forming regions, mid-IR luminosities of galaxies are often used as proxies for their current star formation rates. However, we have shown that the TP-AGB and RSG stars can also contribute to the mid-IR fluxes of galaxies, with the bulk of the mid-IR contribution coming from the rare but extremely dusty $\mathrm{x}$-AGB stars. In the SMC, the contribution of TP-AGB and RSG stars at $8 \mu \mathrm{m}$ is $\approx 19 \%$ ! If these $\mathrm{x}$-AGB stars are neglected in the accounting of the mid-IR light, then an SMC SFR based on $8 \mu \mathrm{m}$ light alone will be biased high.

This result has impacts on the interpretation of thousands of mid-IR based star formation rates, for example at $z=2$, Spitzer
$24 \mu \mathrm{m}$ imaging traces rest-frame $8 \mu \mathrm{m}$, where the TP-AGB is potentially an important flux contributor. However, the size of the effect may be small in practice. For instance, in the LMC, the TP-AGB + RSG $8 \mu \mathrm{m}$ contribution is $<5 \%$. Compared with the SMC, the LMC has a significantly larger contribution from warm dust and $8 \mu \mathrm{m}$ PAH emission (Bot et al. 2010, and Figure 2 here). Samples of the extremely dusty $z=2$ galaxies detected by Spitzer have been shown to have large PAH flux contributions (e.g., Desai et al. 2009), so the correction for TP-AGB stars may be small in the high- $z$ luminous infrared galaxies. However, it is possible that TP-AGB stars artificially elevate the restframe $8 \mu \mathrm{m}$ estimated SFRs of galaxies, especially for lowermetallicity systems like the SMC, where (1) PAH emission is limited, (2) C-AGB stars are more common, and (3) star formation rates are likely smaller.

We are not the first to point out that the TP-AGB may be important at mid-IR wavelengths. For instance, Kelson \& Holden (2010) and Chisari \& Kelson (2012) recently proposed TP-AGB stars as the primary source of mid-IR flux in galaxies, and may account for the rise of luminous infrared galaxies with redshift. Similarly, Verley et al. (2009) suggested that dusty shells surrounding AGB stars could account for the bulk of diffuse $24 \mu \mathrm{m}$ emission in the outskirts of M33. While our results show the importance of TP-AGB stars at mid-IR wavelengths, these earlier works likely overemphasize the TPAGB contribution, especially at $24 \mu \mathrm{m}$, where we show that TP-AGB stars contribute less than $3 \%$ of the Magellanic Cloud flux.

The large mid-IR TP-AGB contributions predicted by these previous works (e.g., $>50 \%$ of the 8 and $24 \mu \mathrm{m}$ flux) likely arose from their adopted mid-IR colors and fluxes of C-AGB stars. Kelson \& Holden (2010) and Chisari \& Kelson (2012) assume that C-rich AGB stars have an average $K-[8.8]$ color of $5.66 \pm 1.16 \mathrm{mag}$, derived from Galactic carbon stars observed in the mid-IR by Guandalini et al. (2006). Verley et al. (2009) assume an average $24 \mu \mathrm{m}$ flux derived from carbon stars observed with Spitzer in the Magellanic Clouds by Groenewegen et al. (2007). However, both the Guandalini et al. (2006) and Groenewegen et al. (2007) samples are heavily biased toward the reddest $\mathrm{x}$-AGB stars and completely exclude the bulk of the C-AGB population, which is much bluer. This issue is demonstrated in the bottom panel of Figure 3, which plots histograms of the $K_{\mathrm{S}}-$ [8] color of the TP-AGB stars in the LMC. The color adopted by the Kelson \& Holden (2010) models is shown as a dashed line and is significantly redder than the bulk of the LMC C-AGB stars. When including the complete samples of C-rich AGB stars (C-AGB plus X-AGB, red and green distributions, respectively) from the SAGE survey, we find $\left\langle K_{\mathrm{S}}-[8]\right\rangle_{\mathrm{x}+\mathrm{C}}=1.38 \mathrm{mag}$ in the $\mathrm{SMC}$ and $1.31 \mathrm{mag}$ in the LMC (triple-dot-dashed line in Figure 3). This is over 4 mag bluer than the Kelson \& Holden models (also used in Chisari \& Kelson 2012). A similarly sized discrepancy is obtained when comparing the SAGE AGB sample to the adopted $24 \mu \mathrm{m}$ flux for AGB stars in Verley et al. These revised colors would result in a much smaller contribution of TP-AGB stars at mid-IR wavelengths, as is seen in Figure 2 for the Magellanic Clouds.

While some $\mathrm{x}$-AGB stars in the SAGE Magellanic Cloud sample do show colors as red as those used in the Kelson $\&$ Holden models, together they have an average color that is still 2 mag bluer than the models, $\left\langle K_{\mathrm{S}}-[8]\right\rangle_{\mathrm{x}}$, SMC $=$ $3.25 \mathrm{mag}$ and $\left\langle K_{\mathrm{S}}-[8]\right\rangle_{\mathrm{x}}$, LMC $=3.41 \mathrm{mag}$ (dot-dashed line in top panel of Figure 3). In addition, these $\mathrm{x}$-AGB stars 
contribute almost nothing to the $K$-band light of the Magellanic clouds ( $1 \%$ or less). The bluer O-AGB and C-AGB stars produce roughly 20 times the $K$-band light of the X-AGB stars. The Kelson \& Holden models rely on the $K$-band flux to estimate the AGB contribution at longer wavelengths, and they assume that the X-AGB stars are contributing nearly $50 \%$ of the $K$-band light. Because of these assumptions, they are likely significantly overestimating the AGB flux contribution at longer wavelengths.

\section{CONCLUSIONS}

The contribution of TP-AGB and RSG stars to the integrated fluxes of the Magellanic Clouds varies with wavelength. We find that their combined contribution peaks between $3-4 \mu \mathrm{m}$, where they contribute $32 \%$ of the SMC flux and $\sim 25 \%$ of the LMC flux. At $1.2 \mu \mathrm{m}$, the contributions are smaller, $\sim 18 \%$ and $\sim 15 \%$ for the SMC and LMC. At longer wavelengths, the contributions also decline, but still constitute $19 \%$ of the $8 \mu \mathrm{m}$ light of the SMC (only 4\% for the LMC where warm dust and PAH emission are stronger). The bulk of this $8 \mu \mathrm{m}$ stellar flux is from the most dust-enshrouded AGB stars, the so-called x-AGB stars, which are predominantly carbon stars near the end of the AGB phase. At $24 \mu \mathrm{m}$, the AGB star contribution to the total flux is less than $3 \%$ for each galaxy.

The flux contributions of the TP-AGB and RSG are significant at $3.6 \mu \mathrm{m}$. Thus, these stars have an impact on the $M / L$ ratios of the Magellanic Clouds at near-IR wavelengths. Because these stars are short-lived, we can expect that the near-IR $M / L$ ratios will also vary significantly over time. To minimize the impact of short lived TP-AGB and RSG stars on stellar mass estimates of galaxies, shorter wavelength observations are preferred. However, at optical wavelengths, short-lived, superluminous $\mathrm{O}$ and B stars play a similar role as the AGB and RSG stars in the near-IR. We find that the optimal wavelengths for minimizing the impact of luminous short-lived stars on the $M / L$ ratio of galaxies is between 0.8 and $1 \mu \mathrm{m}$.

The non-negligible flux from the TP-AGB at $8 \mu \mathrm{m}$ suggests that star formation rates based on rest-frame $8 \mu \mathrm{m}$ flux (e.g., Spitzer $24 \mu \mathrm{m}$ imaging at $z=2$ ) may be biased slightly high. However, this effect is most important for systems with little PAH emission (like the SMC). For systems with significant warm dust and PAH emission, the TP-AGB contribution at $8 \mu \mathrm{m}$ appears to be small, and thus for the LMC the contribution is $<4 \%$.

Special thanks to Karl Gordon for providing integrated fluxes of the LMC in the Spitzer bands, and to Caroline Bot for sharing the SED and model fits of the Magellanic Clouds. Thanks to Gustavo Bruzual for sharing updated single stellar population models of the Magellanic Clouds. We also thank Barry Madore, Mark Seibert, and Wendy Freedman for inspiring efforts to study the impact of AGB stars on mid-infrared galaxy fluxes. This work is based in part on observations made with the Spitzer Space Telescope, which is operated by the Jet Propulsion Laboratory, California Institute of Technology under a contract with NASA.

\section{REFERENCES}

Bell, E. F., \& de Jong, R. S. 2001, ApJ, 550, 212

Bertelli, G., Girardi, L., Marigo, P., \& Nasi, E. 2008, A\&A, 484, 815 Bertelli, G., Nasi, E., Girardi, L., \& Marigo, P. 2009, A\&A, 508, 355 Blum, R. D., Mould, J. R., Olsen, K. A., et al. 2006, AJ, 132, 2034 Bot, C., Ysard, N., Paradis, D., et al. 2010, A\&A, 523, A20

Bowen, G. H. 1988, ApJ, 329, 299

Boyer, M. L., Skillman, E. D., van Loon, J. Th., Gehrz, R. D., \& Woodward, C. E. 2009, ApJ, 697, 1993

Boyer, M. L., Srinivasan, S., van Loon, J. Th., et al. 2011, AJ, 142, 103

Bruzual, A. G. 2011, RMxAC, 40, 36

Chary, R., \& Elbaz, D. 2001, ApJ, 556, 562

Chisari, N. E., \& Kelson, D. D. 2012, ApJ, 753, 94

Cioni, M., Girardi, L., Marigo, P., \& Habing, H. J. 2006, A\&A, 448, 77

Conroy, C., Gunn, J. E., \& White, M. 2009, ApJ, 699, 486

Dalcanton, J. J., Williams, B. F., Melbourne, J. L., et al. 2012, ApJS, 198, 6

Desai, V., Soifer, B. T., Dey, A., et al. 2009, ApJ, 700, 1190

Díaz-Santos, T., Alonso-Herrero, A., Colina, L., et al. 2008, ApJ, 685, 211

Dohm-Palmer, R. C., \& Skillman, E. D. 2002, AJ, 123, 1433

Elitzur, M., \& Ivezić, Ž. 2001, MNRAS, 327, 403

Eskew, M. R., Zaritsky, D. F., \& Meidt, S. E. 2012, AJ, 143, 139

Fazio, G. G., Hora, J. L., Allen, L. E., et al. 2004, ApJS, 154, 10

Gerke, J. R., \& Kochanek, C. S. 2013, ApJ, 762, 64

Girardi, L., Groenewegen, M. A. T., Hatziminaoglou, E., \& da Costa, L. 2005, A\&A, 436, 895

Girardi, L., Williams, B. F., Gilbert, K. M., et al. 2010, ApJ, 724, 1030

Gordon, K. D., Meixner, M., Meade, M. R., et al. 2011, AJ, 142, 102

Groenewegen, M. A. T., Wood, P. R., Sloan, G. C., et al. 2007, MNRAS, 376,313

Guandalini, R., Busso, M., Ciprini, S., Silvestro, G., \& Persi, P. 2006, A\&A, 445,1069

Harris, J., \& Zaritsky, D. 2004, AJ, 127, 1531

Harris, J., \& Zaritsky, D. 2009, AJ, 138, 1243

Hauser, M. G., Arendt, R. G., Kelsall, T., et al. 1998, ApJ, 508, 25

Iben, I., Jr. 1983, ApJL, 275, 65

Ilbert, O., Salvato, M., Le Floc'h, E., et al. 2010, ApJ, 709, 644

Israel, F. P., Wall, W. F., Raban, D., et al. 2010, A\&A, 519, A67

Kelson, D. D., \& Holden, B. P. 2010, ApJL, 713, 28

Maraston, C., Daddi, E., Renzini, A., et al. 2006, ApJ, 652, 85

Marigo, P., \& Girardi, L. 2007, A\&A, 469, 239

Marigo, P., Girardi, L., Bressan, A., et al. 2008, A\&A, 482, 883

Mattsson, L., Wahlin, R., Höfner, S., \& Eriksson, K. 2008, A\&A, 484, L5

McQuinn, K. B. W., Skillman, E. D., Dalcanton, J. J., et al. 2011, ApJ, 740,48

Meidt, S. E., Schinnerer, E., Knapen, J. H., et al. 2012a, ApJ, 744, 17

Meidt, S. E., Schinnerer, E., Muñoz-Mateos, J.-C., et al. 2012b, ApJL, 748, 30

Meixner, M., Gordon, K. D., Indebetouw, R., et al. 2006, AJ, 132, 2268

Melbourne, J., Williams, B. F., Dalcanton, J. J., et al. 2012, ApJ, 748, 47

Reid, N. 1991, ApJ, 382, 143

Rieke, G. H., Young, E. T., Engelbracht, C. W., et al. 2004, ApJS, 154, 25

Schirrmacher, V., Woitke, P., \& Sedlmayr, E. 2003, A\&A, 404, 267

Sedlmayr, E., \& Dominik, C. 1995, SSRv, 73, 211

Sheth, K., Regan, M., Hinz, J. L., et al. 2010, PASP, 122, 1397

Skrutskie, M. F., Cutri, R. M., Stiening, R., et al. 2006, AJ, 131, 1163

van Loon, J. T., Boyer, M. L., \& McDonald, I. 2008, ApJL, 680, 49

van Loon, J. Th., Marshall, J. R., \& Zijlstra, A. A. 2005, A\&A, 442, 597

Verley, S., Corbelli, E., Giovanardi, C., \& Hunt, L. K. 2009, A\&A, 493, 453

Winters, J. M., Le Bertre, T., Jeong, K. S., Helling, C., \& Sedlmayr, E. 2000, A\&A, 361, 641

Winters, J. M., Le Bertre, T., Jeong, K. S., Nyman, L.-Å., \& Epchtein, N. 2003, A\&A, 409, 715

Woitke, P. 2006, A\&A, 452, 537

Woitke, P. 2007, in ASP Conf. Ser. 378, Why Galaxies Care About AGB Stars: Their Importance as Actors and Probes, ed. F. Kerschbaum, C. Charbonnel, \& R. F. Wing (San Francisco, CA: ASP), 156

Zaritsky, D., Harris, J., Thompson, I. B., Grebel, E. K., \& Massey, P. 2002, AJ, 123,855

Zibetti, S., Charlot, S., \& Rix, H.-W. 2009, MNRAS, 400, 1181 